\title{
COVID-19 Vaccines Tolerated in Patients with Paclitaxel and Docetaxel Allergy
}

\author{
Aleena Banerji ${ }^{1}$, Anna R. Wolfson ${ }^{1}$, Lacey B. Robinson ${ }^{1}$, Aubree E. McMahon ${ }^{1}$, Amelia S. \\ Cogan $^{1}$, Rebecca R. Saff ${ }^{1}$, and Kimberly Blumenthal ${ }^{1}$ \\ ${ }^{1}$ Massachusetts General Hospital Division of Rheumatology Allergy and Immunology
}

July 28, 2021

\section{COVID-19 Vaccines Tolerated in Patients with Paclitaxel and Docetaxel Allergy}

\section{To the Editor:}

After initial reports of anaphylaxis to the messenger RNA (mRNA) COVID-19 vaccines, the Centers for Disease Control and Prevention (CDC) put forth guidance stating that patients with a history of anaphylaxis to vaccine components like polyethylene glycol (PEG) should not receive the mRNA COVID-19 vaccines. ${ }^{1}$ To address this clinical challenge and decrease vaccine hesitancy, we published an approach to guide COVID19 vaccination in high-risk allergy individuals. ${ }^{2-4}$ While the etiology of anaphylaxis to mRNA COVID-19 vaccines remains unclear, PEG continues to be an important focus. ${ }^{5}$ Paclitaxel contains polyoxyl-35 castor oil -a PEG derivative and the excipient in Pfizer-BioNTech and Moderna COVID-19 vaccines- and docetaxel contains polysorbate 80 -the excipient in Janssen COVID-19 vaccine. Given this, we sought to assess the utility of pre-vaccine excipient skin testing (ST), risk stratification and COVID-19 vaccine tolerability in oncology patients with a history of paclitaxel or docetaxel hypersensitivity reaction (HSR).

We included consecutive paclitaxel or docetaxel HSR patients referred to allergy/immunology for evaluation prior to COVID-19 vaccination. Evaluation included clinical risk assessment and excipient ST as previously described. ${ }^{2,4}$ Clinical details were obtained by electronic health record (EHR) review. COVID-19 vaccine tolerance was determined by an allergy/immunology physician (AB) using EHR review, phone call, and/or e-mail. This study was approved by the Massachusetts General Brigham Institutional Review Board and deemed minimal risk.

Between December 30, 2020 and April 2, 2021, 21 patients with paclitaxel $(\mathrm{n}=17)$ or docetaxel $(\mathrm{n}=4)$ HSR were referred (Table 1 ). Most were female $(n=20,95 \%)$ and white $(n=20,95 \%)$. Approximately half of HSRs occurred in the past 4 years $(\mathrm{n}=11,52 \%)$ and $6(29 \%)$ HSRs were anaphylactic. 20 patients $(95 \%)$ had negative excipient ST. One paclitaxel HSR patient had positive ST (methyl-prednisolone acetate PEG3350, $0.4 \mathrm{mg} / \mathrm{mL}$ intradermal) and tolerated the Janssen COVID-19 vaccine (Table 2 ). All patients completed COVID-19 vaccination with no reaction $(\mathrm{n}=19,90 \%)$ or with mild symptoms treated with antihistamines alone $(\mathrm{n}=2,10 \%)$. Of 17 patients with history of paclitaxel HSR, 12 (71\%) received an mRNA COVID-19 vaccine and none had a reaction.

This case series of 21 patients suggests that patients with paclitaxel (containing the excipient polyoxyl-35 castor oil/PEG derivative) or docetaxel (containing the excipient polysorbate 80) HSRs tolerate COVID-19 vaccination. While 2 patients (10\%) developed reactions after COVID-19 vaccination, symptoms resolved with antihistamines alone. The reactions do not appear related to the patient's specific excipient allergy history (e.g., docetaxel HSR patient developed reaction with mRNA vaccine) and in fact, no reactions occurred in paclitaxel HSR patients that received an mRNA COVID-19 vaccine. It remains unclear if the paclitaxel HSR patient with positive intradermal PEG ST would have tolerated mRNA COVID-19 
vaccination, but experience to date indicates that positive PEG intradermal ST does not predict reactions to mRNA COVID-19 vaccines. ${ }^{4}$

CDC guidance advises patients with PEG allergy to proceed with Janssen COVID-19 vaccination while patients with polysorbate 80 allergy can proceed with mRNA COVID-19 vaccines. ${ }^{1}$ However, if both COVID19 vaccine platforms are not routinely accessible, allergy/immunology consultation may be useful for allergy risk assessment and vaccine guidance. Furthermore, if mRNA COVID-19 vaccines are proven optimal for oncology patients, allergy assessment and limited excipient ST (e.g. Miralax, PEG- 3350) ${ }^{2,4}$ can provide reassurance prior to mRNA COVID-19 vaccination in patients with HSR to chemotherapeutics containing PEG. We continue to advise 30-minute monitoring post COVID-19 vaccination for all patients with any history of anaphylaxis per CDC guidance. ${ }^{1}$ This study was limited by its small sample from a single institution and retrospective study design.

The role of excipient ST prior to COVID-19 vaccination appears limited and patients largely tolerated COVID-19 vaccination despite paclitaxel or docetaxel HSR. Reassuringly, COVID-19 vaccine anaphylaxis remains rare.

\section{REFERENCES}

1. Information about COVID-19 Vaccines for People with Allergies. Atlanta, GA: Center for Disease Control and Prevention; 2021. Available at: https://www.cdc.gov/coronavirus/2019ncov/vaccines/recommendations/specific-groups/allergies.html Accessed June 21, 2021.

2. Banerji A, Wickner PG, Saff R, Stone CA Jr, Robinson LB, Long AA, Wolfson AR, Williams P, Khan DA, Phillips E, Blumenthal KG. mRNA Vaccines to Prevent COVID-19 Disease and Reported Allergic Reactions: Current Evidence and Suggested Approach. J Allergy Clin Immunol Pract. 2021 Apr;9(4):1423-1437. doi: 10.1016/j.jaip.2020.12.047. Epub 2020 Dec 31.

3. Banerji A, Wolfson AR, Wickner PG, Cogan AS, McMahon AE, Saff R, Robinson LB, Phillips E, Blumenthal KG. COVID-19 Vaccination in Patients with Reported Allergic Reactions: Updated Evidence and Suggested Approach. J Allergy Clin Immunol Pract. 2021 Jun;9(6):2135-2138.

4. Wolfson AR, Robinson LB, Li L, McMahon AE, Cogan AS, Fu X, Wickner P, Samarakoon U, Saff RR, Blumenthal KG, Banerji A. First Dose mRNA COVID-19 Vaccine Allergic Reactions: Limited Role for Excipient Skin Testing. J Allergy Clin Immunol Pract. 2021 Jun 21:S2213-2198(21)00675-9.

5. Sellaturay P, Nasser SM, Islam S, Gurugama P, Ewan PW. Polyethylene glycol (PEG) is a cause of anaphylaxis to the Pfizer/BioNTech mRNA COVID-19 vaccine. Clin Exp Allergy. 2021, April 6.

\section{Author names, Affiliations, Funding Sources and Conflict of Interest}

Aleena Banerji, $\mathrm{MD}^{1}$

Anna R. Wolfson, $\mathrm{MD}^{1}$

Lacey B. Robinson, MD, MPH ${ }^{1}$

Aubree E. McMahon, BA ${ }^{1}$

Amelia S. Cogan, $\mathrm{MPH}^{1}$

Rebecca R. Saff, MD, $\mathrm{PhD}^{1}$

Kimberly G. Blumenthal, MD, MSc ${ }^{1,2}$

${ }^{1}$ Division of Rheumatology, Allergy, and Immunology, Department of Medicine, Massachusetts General Hospital, Boston, MA

${ }^{2}$ Edward P. Lawrence Center for Quality and Safety, Massachusetts General Hospital, Boston, MA 
Corresponding Author: Aleena Banerji, MD, Massachusetts General Hospital, Allergy and Immunology, Cox 201, 55 Fruit Street, Boston, MA 02114, p-(617) 726-3850, f- (617) 724-0239, abanerji@mgh.harvard.edu; Twitter: @AleenaBanerjiMD

Funding: None

\section{Conflict of Interest: None}

Word Count: 600

Tables: 2

\section{Hosted file}

PaclitaxelDocetaxel_TablesandFigures FINAL.docx available at https://authorea.com/users/ 427995/articles/532031-covid-19-vaccines-tolerated-in-patients-with-paclitaxel-anddocetaxel-allergy 